\title{
Pre-treatment of sperm reduces success of ICSI in the pig
}

\author{
Michiko Nakai, Junya Ito ${ }^{1}$, Ken-ichi Sato ${ }^{2}$, Junko Noguchi, Hiroyuki Kaneko, Naomi Kashiwazaki ${ }^{1}$ \\ and Kazuhiro Kikuchi
}

\begin{abstract}
Reproductive Biology Research Unit, Division of Animal Sciences, National Institute of Agrobiological Sciences, Tsukuba, Ibaraki 305-8602, Japan, ' Laboratory of Animal Reproduction, School of Veterinary Medicine, Azabu University, Sagamihara, Kanagawa 252-5201, Japan and ${ }^{2}$ Department of Molecular Biosciences, Faculty of Life Sciences, Kyoto Sangyo University, Kyoto, Kyoto 603-8555, Japan
\end{abstract}

Correspondence should be addressed to M Nakai; Email: nakai3@affrc.go.jp

\begin{abstract}
In pigs, although ICSI is a feasible fertilization technique, its efficiency is low. In general, injected pig sperm are insufficient to induce oocyte activation and embryonic development. Pretreatments for disrupting sperm membranes have been applied to improve the fertility of ICSI oocytes; however, we hypothesize that such pretreatment(s) may reduce the ability of the sperm to induce oocyte activation. We first evaluated the effects of sperm pretreatments (sonication (SO) to isolate the sperm heads from the tails, Triton X-100 (TX), and three cycles of repeated freezing/thawing $(3 \times-\mathrm{FT})$ for disrupting sperm membranes) on the rate of pronucleus (PN) formation after ICSI. We found that oocytes injected with control (whole) sperm had higher rates of PN formation than those obtained after subjecting the sperm

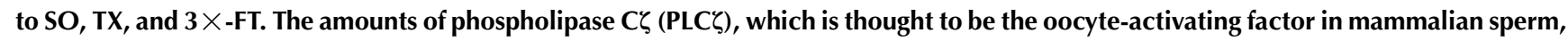
in sperm treated by each method was significantly lower than that in whole untreated sperm. Furthermore, using immunofluorescence, it was found that in pig sperm, PLC $\zeta$ was localized to both the post-acrosomal region and the tail area. Thus we demonstrated for the first time that sperm pretreatment leads to a reduction of oocyte-activating capacity. Our data also show that in addition to its expected localization to the sperm head, PLC $\zeta$ is also localized in the tail of pig sperm, thus raising the possibility that injection of whole sperm may be required to attain successful activation in pigs.

Reproduction (2011) 142 285-293
\end{abstract}

\section{Introduction}

Ovulated mammalian oocytes remain arrested until fertilization at the metaphase of the second meiosis (metaphase-II). In mammals, repetitive rises in the intracellular level of free $\mathrm{Ca}^{2+}$, also known as $\mathrm{Ca}^{2+}$ oscillations, are induced immediately after spermoocyte fusion, which is a prerequisite event for oocyte activation (Miyazaki \& Ito 2006). Research has indicated that phospholipase $\mathrm{C} \zeta$ (PLC) ) might be the sperm factor that triggers $\mathrm{Ca}^{2+}$ responses during fertilization in fish (Ito et al. 2008), rodents (Saunders et al. 2002, Ito et al. 2008), birds (Coward et al. 2005, Mizushima et al. 2009), primates (Cox et al. 2002), and also domestic species (cattle (Ross et al. 2008) and pigs (Yoneda et al. 2006)). The amplitude and number of rises of $\mathrm{Ca}^{2+}$ are suggested to play an important role in the completion of the events of oocyte activation, including the regulation of maternally stored mRNA in the early zygote (Ducibella et al. 2002), which might affect gene expression at later stages of preimplantation embryo development (Ozil et al. 2006) as well as cell composition in blastocysts (Bos-Mikich et al. 1997).
ICSI has been used to generate offspring in infertile human couples and also in experimental and domestic animals (Yanagimachi 2005). In studies using rodent sperm, injection of whole sperm (mouse (Kimura \& Yanagimachi 1995, Kuretake et al. 1996)) or of a sperm head (mouse (Kuretake et al. 1996), rat (Hirabayashi et al. 2002, Nakai et al. 2005), and hamster (Yamauchi et al. 2002)) is sufficient to generate full-term development. These results suggest that in these species, a large part of the activating stimulus resides in the sperm head.

In large animals, the birth of live offspring after ICSI has been reported (pigs (Kolbe \& Holtz 2000, Martin 2000, Nakai et al. 2003), cattle (Goto et al. 1990); sheep (Catt et al. 1996), and horses (Cochran et al. 1998)), although it is well known that developmental success in these species remains remarkably low after this technique. In natural fertilization, oocytes penetrated by spermatozoa are activated normally (as evidenced by extrusion of the second polar body and formation of the male and female pronuclei), but the efficiency of oocyte activation after ICSI is quite low unless the oocytes further receive artificial stimulation (Fujinami et al. 2004, 
Table 1 The effects of sperm treatments on pronuclear formation after ICSI.

\begin{tabular}{|c|c|c|c|c|}
\hline \multirow[b]{2}{*}{ Sperm treatments ${ }^{a}$} & \multirow{2}{*}{$\begin{array}{l}\text { Number of the injected } \\
\text { oocytes }(N)\end{array}$} & \multicolumn{3}{|c|}{ Number of pronuclear formed oocytes ( $\%$ from $N$ ) } \\
\hline & & Total & Normal fertilization ${ }^{b}$ & Others $^{\mathrm{c}}$ \\
\hline Whole & 79 & $43(54.4 \pm 4.5)^{*}$ & $33(41.8 \pm 7.4)^{*}$ & $10(12.6 \pm 6.5)$ \\
\hline $\mathrm{SO}$ & 59 & $12(20.3 \pm 3.6)^{\dagger}$ & $8(13.5 \pm 0.8)^{\dagger}$ & $4(6.8 \pm 3.2)$ \\
\hline $\mathrm{TX}$ & 74 & $7(9.5 \pm 5.8)^{\dagger}$ & $4(5.4 \pm 3.8)^{t}$ & $3(4.1 \pm 3.9)$ \\
\hline $3 \times-F T$ & 64 & $14(21.9 \pm 5.0)^{\dagger}$ & $9(14.1 \pm 5.3)^{\dagger}$ & $5(7.8 \pm 3.0)$ \\
\hline Sham & 69 & $6(8.7 \pm 2.7)^{t}$ & $0(0)^{+}$ & $6(8.7 \pm 2.7)$ \\
\hline Handling & 82 & $1(1.2 \pm 0.9)^{\dagger}$ & $0(0)^{+}$ & $1(1.2 \pm 0.9)$ \\
\hline
\end{tabular}

Data are presented as mean \pm S.E.M. of more than three separate replicates. Different superscripts within each column $(*,+)$ indicate values that are significantly different $(P<0.05)$.

${ }^{a}$ Frozen-thawed spermatozoa (whole) were treated with sonication (SO), $0.1 \%$ Triton X-100 (TX), or freezing/thawing without cryoprotectant three

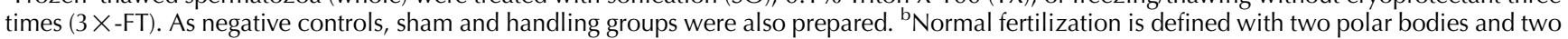
pronuclei. ${ }^{\mathrm{C}}$ Others are categorized other than normal fertilization.

Nakai et al. 2006). Therefore, it is suspected that delivery of the oocyte activation signal by the sperm in large domestic species may be compromised.

Various artificial stimulation protocols, such as electrical stimulation (Probst \& Rath 2003, Nakai et al. 2006), application of calcium ionophore (Kolbe \& Holtz 1999, Probst \& Rath 2003) or ionomycin (Galli et al. 2003), and combined treatment with ionomycin and 6-dimethylaminopurine (Gou et al. 2002, JimenezMacedo et al. 2005), ethanol (Fujinami et al. 2004), or $\mathrm{CaCl}_{2}$ (Probst \& Rath 2003), have been used to improve ICSI in domestic species. Although these protocols improve pronucleus (PN) formation and embryonic development, the subsequent developmental rates do not reach those observed after IVF, at least in pigs (Kikuchi et al. 2002, Nakai et al. 2003, 2006).

In pig oocytes, failure of male $\mathrm{PN}$ formation frequently occurs after ICSI, even though female PN formation seems to occur normally (Kren et al. 2003, Lee et al. 2003). To improve male PN formation, various treatments of sperm have been applied to sperm such as sonication (SO; Kim et al. 1999), incubation with TritonX 100 (TX; Lee \& Yang. 2004), exposure to progesterone (Katayama et al. 2002), or repetitive freezing/thawing (FT) without cryoprotectants (Katayama et al. 2002). However, our studies using some of these procedures have shown that the rates of male PN formation are still remarkably low (Nakai et al. 2006). Therefore, we have hypothesized that such treatments may reduce the ability of pig sperm to induce oocyte activation. In this study, we investigated the effects of several sperm treatments on the concentration and localization of $\mathrm{PLC} \zeta$ in pig sperm and on the ability of these sperm to induce oocyte activation.

\section{Results}

\section{Treatment of sperm before ICSI affects PN formation}

The ability of pretreated sperm to induce PN formation after ICSI was examined (Table 1). The percentage of PN formation in the SO $(20.3 \pm 3.6 \%), \operatorname{TX}(9.5 \pm 5.8 \%)$, and three cycles of repeated freezing/thawing $(3 \times-\mathrm{FT}$; $21.9 \pm 5.0 \%)$ treatment groups was lower $(P<0.05)$ than that for oocytes injected with whole sperm $(54.4 \pm 4.5 \%)$ and in fact was as low as that after sham injection $(8.7 \pm 2.7 \%)$ or dummy handling $(1.2 \pm 0.9 \%)$. In addition, the proportion of normally fertilized zygotes among oocytes injected with whole sperm (41.8 $\pm 7.4 \%)$ was significantly $(P<0.05)$ higher than those in the other groups (SO (13.5 $\pm 0.8 \%)$, TX $(5.4 \pm 3.8 \%)$, and $3 \times-\mathrm{FT}$ $(14.1 \pm 5.3 \%))$.

We next examined whether injection of multiple sonicated sperm heads would be able to induce higher rates of $\mathrm{PN}$ formation. When one sperm head was injected, more than one $\mathrm{PN}$ formation rate was low $(20.4 \pm 3.6 \%$; Table 2). However, as the number of sperm heads increased, the rates of oocyte with more than one $\mathrm{PN}$ also increased, reaching $78.0 \pm 7.5 \%$ when three sperm heads were injected.

Table 2 Improvement of oocyte activation by injection of multiple sperm heads.

\begin{tabular}{|c|c|c|c|c|c|c|c|c|c|c|}
\hline \multirow[b]{2}{*}{ Group $^{a}$} & \multirow{2}{*}{$\begin{array}{l}\text { Number of } \\
\text { oocytes }\end{array}$} & \multicolumn{4}{|c|}{ Oocytes with first polar body } & \multicolumn{4}{|c|}{ Oocytes with two polar bodies } & \multirow[b]{2}{*}{$1 \mathbf{P N}<(\%)$} \\
\hline & & $1 \mathrm{PN}(\%)$ & $2 \mathrm{PN}(\%)$ & $3 \mathrm{PN}(\%)$ & $4 \mathrm{PN}(\%)$ & $1 \mathrm{PN}(\%)$ & $2 \mathrm{PN}(\%)$ & $3 \mathrm{PN}(\%)$ & $4 \mathrm{PN}(\%)$ & \\
\hline Head-1 & 59 & $1(1.7 \pm 1.9)$ & $1(1.7 \pm 1.9)$ & $0(0)$ & $0(0)$ & $2(3.4 \pm 1.5)$ & $8(13.6 \pm 0.7)$ & $0(0)$ & $0(0)$ & $12(20.3 \pm 3.6)^{*}$ \\
\hline Head-2 & 52 & $0(0)$ & $0(0)$ & $2(3.8 \pm 2.4)$ & $1(1.9 \pm 3.3)$ & $1(1.9 \pm 1.3)$ & $4(7.7 \pm 4)$ & $10(19.2 \pm 3.9)$ & $1(1.9 \pm 2)$ & $19(36.5 \pm 5.2)^{*}$ \\
\hline Head-3 & 50 & $1(2.0 \pm 3.3)$ & $0(0)$ & $1(2.0 \pm 3.3)$ & $1(2.0 \pm 3.3)$ & $5(10.0 \pm 5.2)$ & $6(12.0 \pm 3.8)$ & $12(48 \pm 5.8)$ & $13(26.0 \pm 8.4)$ & $39(78.0 \pm 7.5)^{\dagger}$ \\
\hline
\end{tabular}

Data are presented as mean \pm S.E.M. of more than three separate experiments. Different superscripts $(*,+)$ indicate values that are significantly different $(P<0.05)$.

${ }^{\mathrm{a}}$ One (head-1), two (head-2), or three (head-3) sonicated sperm head(s) were injected into oocytes. 

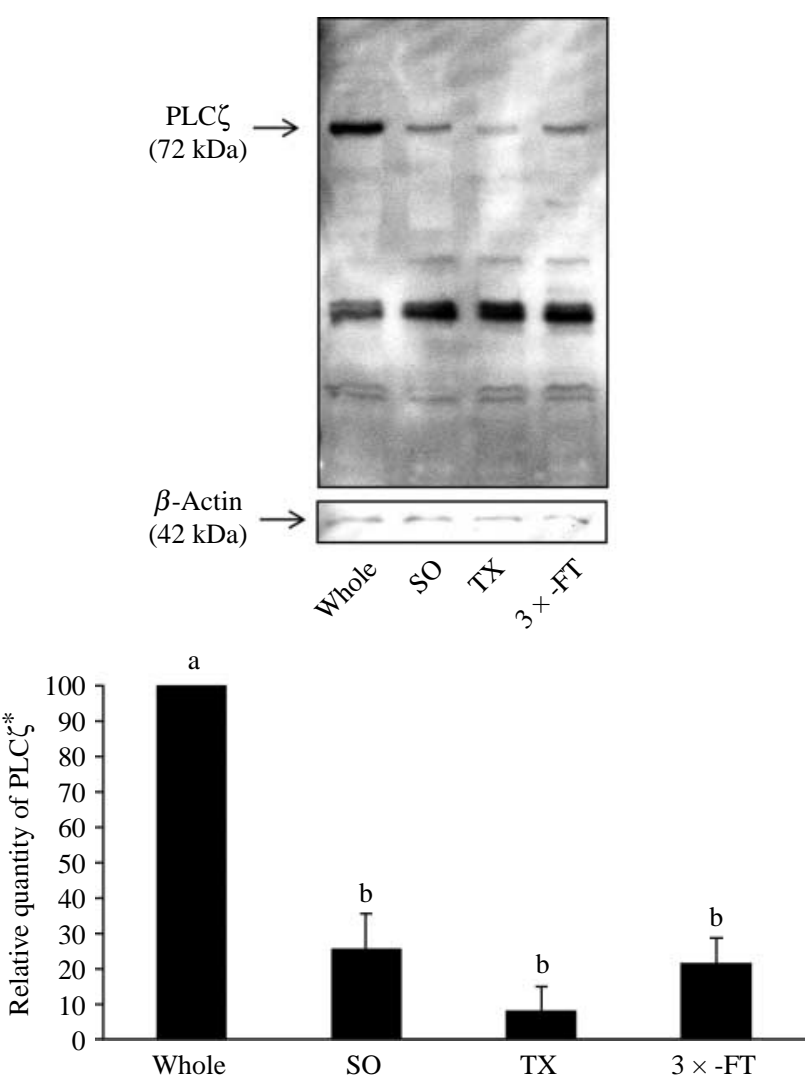

Figure 1 Decrease in the quantity of PLC $\zeta$ in pig spermatozoa after demembrane treatments. Frozen-thawed spermatozoa were treated with sonication (SO), $0.1 \%$ Triton X-100 (TX), or freezing/thawing without cryoprotectant three times $(3 \times-\mathrm{FT})$. Western blot analysis is shown in the upper panels and the mean values of band intensities in the lower panel. Blotting with anti- $\beta$ actin antibody is also shown as a protein loading control. The concentration of sperm was $1 \times 10^{5}$ cells/lane. *Data are expressed in terms of the fold strength of the PLC $\zeta$ level in the whole group and are presented as mean \pm S.E.M. for three separate experiments. Different letters $(a, b)$ indicate values that are significantly different $(P<0.05)$.

\section{PLC concentration and distribution are altered after sperm treatments}

We next examined how the aforementioned treatments affected the concentration of PLC $\zeta$. The concentrations of PLC $\zeta$ in the SO, TX, and $3 \times-F T$ groups were markedly reduced in comparison with that in the whole group $(P<0.05 ;$ Fig. 1$)$, although all the treatments induced the same degree of PLC $\zeta$ loss (Fig. 1).

Furthermore, we investigated the localization of PLC in boar sperm before and after each sperm treatment (Fig. 2). A strong immunoreactive signal was observed in the acrosomal and post-acrosomal regions of whole sperm, which were used as positive controls (Fig. 2A, A'). In addition, the tail region, especially around the neck, also showed a faint fluorescent signal (Fig. 2A, $A^{\prime}$ ). To confirm whether the signals were specific to PLC $\zeta$, samples were pretreated with an antigenic peptide. As a result, the signal in the post-acrosomal and tail regions disappeared, but the signal localized to the acrosomal area did not (Fig. 2B, $\mathrm{B}^{\prime}$ ). In the absence of primary antibody, the fluorescence for PLC $\zeta$ was not detectable (Fig. 2C, $\mathrm{C}^{\prime}$ ). Importantly, all of the aforementioned treatments caused the immunoreactive area around the post-acrosomal region to disappear (TX: Fig. 2D, $\mathrm{D}^{\prime}, 3 \times-\mathrm{FT}$ : Fig. $2 \mathrm{E}, \mathrm{E}^{\prime}$, and SO: Fig. $2 \mathrm{~F}, \mathrm{~F}^{\prime}$ ).

\section{PLCS detection in supernatant after treatment of pig sperm}

Given the reduced presence of PLC $\zeta$ after the treatments, we speculated that PLC $\zeta$ might be released into the surrounding medium. Figure 3 shows that this was indeed the case, as western blot analysis detected a much greater amount of immunoreactive PLC $\zeta$ in the supernatant from the $\mathrm{SO}, \mathrm{TX}$, and $3 \times$-FT groups than in that from whole untreated sperm.

\section{Sperm tail also participates in induction of oocyte activation}

Given the presence of PLC $\zeta$ in the sperm tail, suggested by our immunofluorescence studies, we evaluated
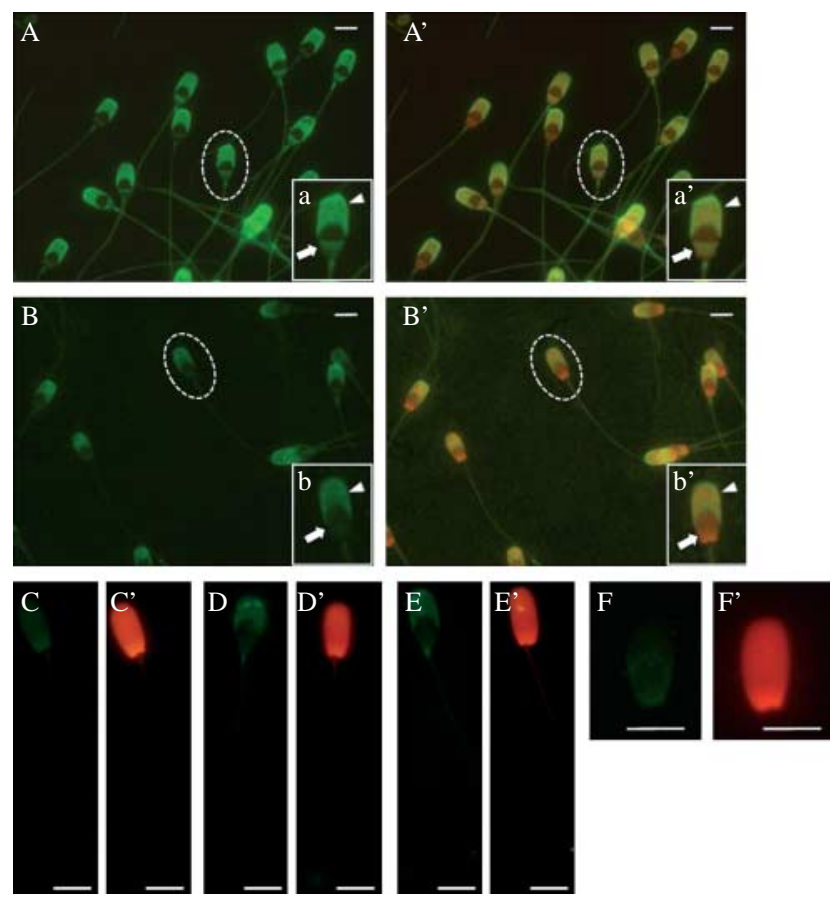

Figure 2 Immunofluorescence analysis of PLC $\zeta$ localization in pig spermatozoa treated with $0.1 \%$ TritonX-100 $\left(D, D^{\prime}\right)$, repetitive freezing/thawing without cryoprotectant three times $\left(E, E^{\prime}\right)$ and sonication $\left(F, F^{\prime}\right)$, or without each treatment respectively $\left(A, A^{\prime}, B, B^{\prime}, C, C^{\prime}\right)$. Labeling of pig sperm with antibody against PLC $\zeta$ (green: $A, B, C, D, E$, F) and combined with $\mathrm{PI}$ (red: $\mathrm{A}^{\prime}, \mathrm{B}^{\prime}, \mathrm{C}^{\prime}, \mathrm{D}^{\prime}, \mathrm{E}^{\prime}, \mathrm{F}^{\prime}$ ). Negative controls were incubated in the absence of primary antibody $\left(C, C^{\prime}\right)$ or with antigenic peptide $\left(10 \mathrm{ng} / \mu \mathrm{l} ; \mathrm{B}, \mathrm{B}^{\prime}\right)$. Magnified views of sperm (identified as circles in $\left.A, A^{\prime}, B, B^{\prime}\right)$ are also shown $\left(a, a^{\prime}, b, b^{\prime}\right)$. Arrows and arrowheads indicate the equatorial and acrosomal regions, respectively. Scale bars: $5 \mu \mathrm{m}$. 


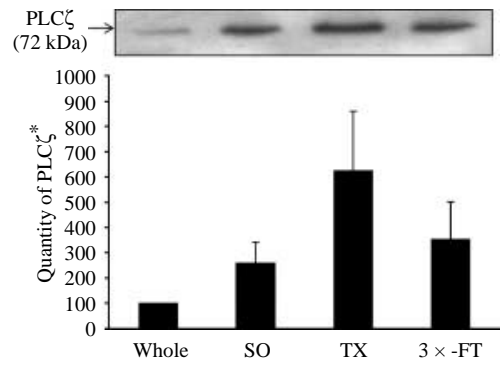

Figure 3 Detection of PLC $\zeta$ in supernatants of sperm cells subjected to different treatments. The amount of PLC $\zeta$ loaded was the PLC $\zeta$ released into the surrounding medium from $5 \times 10^{5}$ cells immediately after each treatment. Frozen-thawed spermatozoa (whole) were treated with sonication (SO), $0.1 \%$ Triton X-100 (TX) or F/T without cryoprotectant three times $(3 \times-\mathrm{FT}) .{ }^{*}$ Data are expressed in terms of the fold strength of the PLC $\zeta$ level in supernatants from whole sperm and are presented as mean \pm s.E.M. for three separate experiments.

whether injection of sperm tails would be able to induce oocyte activation events (Fig. 4). The following injection groups were prepared: 1 ) one sperm head (head group), 2) one sperm tail (1-tail group), 3) three sperm tail (3-tail group), 4) one sperm head and one tail (head+tail group), and 5) sham injection (sham group). The rates of metaphase-II-arrested oocytes in the head (64.4 $\pm 7.9 \%)$, 1 -tail $(64.4 \pm 2.7 \%)$, 3-tail $(51.3 \pm 7.2 \%)$, and head + tail $(29.6 \pm 5.8 \%)$ groups were lower than those in the sham group $(89.9 \pm 2.8 \% ; P<0.05)$. In addition, the rate of $\mathrm{PN}$ formation in oocytes injected with head+tail (40.7 $\pm 6.2 \%)$ was higher than that in the other groups $(P<0.05)$. There were no significant differences in the proportions of oocytes showing $\mathrm{PN}$ formation among the head $(20.3 \pm 3.6 \%)$, 1-tail $(3.4 \pm 3.6 \%)$, 3 -tail $(20.5 \pm 2.7 \%)$, and sham $(8.7 \pm 2.7 \%)$ groups. However, there found to be a tendency of difference ( $P=0.085)$ between 1-tail and 3-tail groups after the analysis used in this study. The proportions of oocytes in the transitional stage in the 1-tail $(32.2 \pm 4.3 \%)$, 3-tail $(25.6 \pm 5.7 \%)$ and head + tail $(29.6 \pm 8.2 \%)$ groups were significantly higher than those in the sham group $(1.4 \pm 1.7 \% ; P<0.05)$. Furthermore, we confirmed the presence of PLC $\zeta$ in sperm tails using western blotting (Fig. 5).

\section{Discussion}

In all domestic animals tested to date, including pigs, the developmental competence of zygotes generated by the ICSI technique has been low in comparison to those obtained using the same procedure in rodents (Kimura \& Yanagimachi 1995, Kuretake et al. 1996, Garcia-Rosello et al. 2009). We hypothesized that the problem may lie in an insufficient oocyte activation stimulus generated by the injected sperm. Initially, therefore, we investigated the membrane-damaging effects of certain treatments on the ability of sperm to induce PN formation after ICSI; some of these treatments are applied to assist decondensation of the sperm head (Lee \& Yang 2004). We then also noted that the rates of PN formation in oocytes injected with treated sperm were lower than those obtained after injection of whole sperm. Similar results have already been reported following treatment of sperm by FT without cryoprotectants or dithiothreitol, although the underlying mechanisms were not investigated (Yong et al. 2005). In addition, we found that injection of multiple sperm heads improved the rates of $\mathrm{PN}$ formation (Table 2). Together, we considered these results to indicate that the $\mathrm{PLC} \zeta$ concentration/activity in sperm may be decreased by sperm treatments that are commonly used prior to ICSI in large domestic species.

To explore the possibility that PLC $\zeta$ had been lost from the sperm, we performed western blotting on supernatants of the sperm preparations and immunoreactive PLC $\zeta$ was indeed detected in these samples (Fig. 3). This was not unexpected, as characterizations of the sperm factor have been reported previously using samples of supernatants obtained from sonicated pig sperm (Kuretake et al. 1996). Furthermore, we observed a reduction of immunofluorescent signals for PLC $\zeta$ in sperm that had undergone the aforementioned treatments (Fig. 2). These findings demonstrated that treatment of pig sperm leads to a reduced ability to induce oocyte activation and that this should be taken into consideration when preparing samples for ICSI (Table 1). Remarkably, in rats, the presence of $\mathrm{PLC} \zeta$ in the head region has been shown to be maintained even after SO and demembranation treatments (Seita et al. 2009). Consistent with these findings, the ability of rat sperm to induce oocyte activation and full-term development following SO and demembranation was not diminished by some of the aforementioned

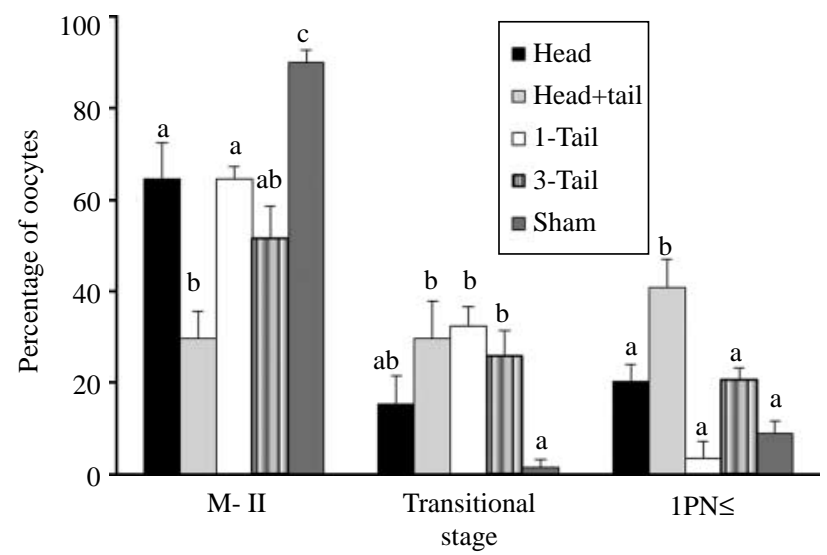

Figure 4 Participation of sperm tails in the induction of oocyte activation. Oocytes were injected with one sonicated sperm head (head), one sonicated sperm tail (1-tail), three sonicated sperm tail (3-tail), or a head and a tail (head+tail). Sham-injected oocytes were prepared as a negative control. At $10 \mathrm{~h}$, oocytes were classified into the following three categories: 1 ) M-II, metaphase-II stage; 2 ) transitional period: total of anaphase-II, telophase-II, and metaphase-III stages; and 3) $1 \mathrm{PN} \leq$ : formation of more than one pronucleus. Data are presented as mean \pm S.E.M. for more than three separate experiments. Different superscripts $(a, b, c)$ within each category indicate values that are significantly different $(P<0.05)$. 


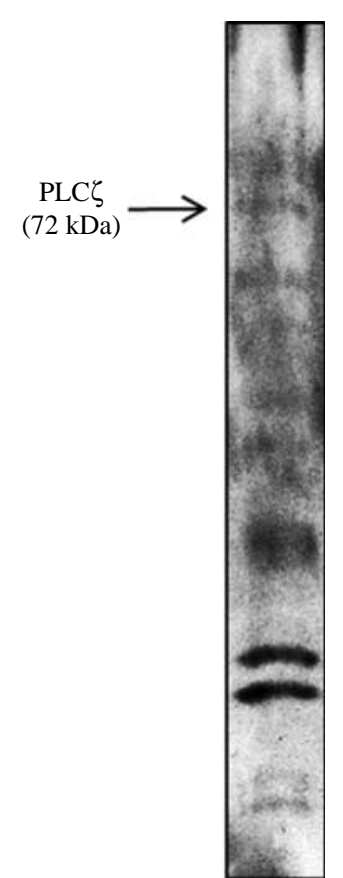

Figure 5 Sperm tails also contain PLC $\zeta$. A band of $\sim 72 \mathrm{kDa}$ was detected in the sperm tail sample. The concentration of sperm tails was $4 \times 10^{6}$ cells/lane.

treatments (Seita et al. 2009). Similarly, sonicated mouse sperm heads still seem to retain their ability to induce oocyte activation (Yazawa et al. 2009). Therefore it seems that the sperm of various mammalian species differ in their PLC $\zeta$ content and/or storage site.

It has been reported that PLC $\zeta$ is detectable in the post-acrosomal region (Yoon \& Fissore 2007) and in the acrosomal and post-acrosomal regions (Young et al. 2009) of mouse sperm, in the acrosomal region of rat sperm (Seita et al. 2009), in the equatorial (Yoon et al. 2008), or equatorial and post-acrosomal regions (Heytens et al. 2009) of human sperm, and in the equatorial region of bull sperm (Yoon \& Fissore 2007). In mice, it is known that the components of the tail are not required for induction of oocyte activation (Kuretake et al. 1996). However, subsequent to the submission of this manuscript, the existence of PLC $\zeta$ in equine sperm tail was suggested (Bedford-Guaus et al. 2011). In this study, we also suggest that PLC $\zeta$ was detectable in both the post-acrosomal region and the tail region of pig sperm (Figs 2 and 5), suggesting that pig sperm tails may be capable of triggering oocyte activation and embryo development. Our results showed that a higher proportion of oocytes injected with sperm tails resumed the second meiosis than sham-injected oocytes, although most of them failed to reach the PN stage. These results suggest that pig sperm tails possess PLC $\zeta$ activity but that its concentration and/or activity is insufficient for PN formation. Indeed, combined injection of a head and a tail greatly improved the rates of metaphase-II exit and $\mathrm{PN}$ formation (Fig. 4). Interestingly, despite the presence of PLC $\zeta$ in both the head and the tail, injection of whole sperm induced normal fertilization in only $40 \%$ of the oocytes (Table 1) and similar results have been published by others (Katayama et al. 2005). In contrast, following IVF, nearly $100 \%$ of sperm-penetrated oocytes display activation events resulting in PN formation (Kikuchi et al. 1999). This suggests that ICSI in these species does not quite replicate the events of fertilization. There are two possible explanations for why this might be the case. First, it is well documented that high rates of polyspermy occur following IVF in the pig (Nagai 1994), which might explain the higher activation rates following this procedure. Alternatively, as we found that an appreciable proportion of pig sperm stained for PLC $\zeta$ did not show any immunoreactivity (data not shown), some of the sperm used for ICSI may lack the enzyme and therefore fail to robustly activate oocytes.

Taken together, our present findings show that treatments used to aid sperm head decondensation before ICSI in the pig also cause loss of PLC $\zeta$, resulting in a weakened sperm activation signal. We have also showed for the first time that PLC $\zeta$ is localized in the post-acrosomal and tail regions of pig sperm and that sperm tails contribute to oocyte activation, although PLC $\zeta$ in the tail region does not represent the main activation stimulus. It is hoped that these findings, and a better understanding of the early stages of oocyte activation, will improve the efficiency of ICSI in the pig and other large domestic species.

\section{Materials and Methods}

Protocols for the use of animals were approved by the Animal Care Committee of the National Institute of Agrobiological Sciences, Japan.

\section{Sperm collection, treatment, and preparation}

Ejaculated semen was collected from boars of the Landrace breed, and cryopreservation of the sperm was carried out as described previously (Kikuchi et al. 1998, Ikeda et al. 2002). Cryopreserved spermatozoa were thawed in TCM 199 (with Earl's salts; Gibco Life Technologies) adjusted to $\mathrm{pH} 7.8$ and centrifuged for $2 \mathrm{~min}$ at $600 \mathrm{~g}$. The sperm pellet was resuspended in pig-fertilization medium (Pig-FM; Suzuki et al. 2002) supplemented with $5 \mathrm{mg} / \mathrm{ml}$ BSA (Fraction V; Sigma-Aldrich Corp.). Sperm (whole group) was then subjected to the following three treatments: 1 ) SO at room temperature for $10 \mathrm{~s}$ using an ultrasonic cleaner (UT-305; Sharp Manufacturing Systems Corporation, Osaka, Japan) to isolate the sperm heads from the tails (SO group), 2) repetitive FT without cryoprotectant three times at $-196{ }^{\circ} \mathrm{C}$ and $37^{\circ} \mathrm{C}$ respectively ( $3 \times$-FT group), and 3 ) incubation in Pig-FM supplemented with $0.1 \%$ TX (Sigma) at $37^{\circ} \mathrm{C}$ for $1 \mathrm{~min}$ (TX group). The treated sperm were centrifuged for $2 \mathrm{~min}$ at $600 \mathrm{~g}$ and the sperm pellets were resuspended in Pig-FM for injection. Injection was conducted within 30 min after each sperm treatment. For 
western blotting analysis, the treated spermatozoa were centrifuged for 5 min at $1400 \mathrm{~g}$ in PBS (Nissui Pharmaceutical Co., Tokyo, Japan) three times. The pellets were then diluted in Laemmli sample buffer (Bio-Rad Laboratories) and stored at $-20^{\circ} \mathrm{C}$.

\section{Preparation of supernatants from treated sperm}

For collection of supernatants, all sperm treatments were conducted in PBS containing protease inhibitors such as $0.2 \mathrm{mM}$ phenylmethylsulfonylfluoride (Sigma), $10 \mu \mathrm{g} / \mathrm{ml}$ pepstatin A (Wako Pure Chemical Industries, Osaka, Japan), $10 \mu \mathrm{g} / \mathrm{ml}$ leupeptin (Wako), $1 \mathrm{mM}$ ethylenediamine$N, N, N^{\prime}, N^{\prime}$-tetraacetic acid (Dojindo Laboratories, Kumamoto, Japan), $10 \mathrm{mM} \beta$-glycerophosphate (Sigma), and $1 \mathrm{mM}$ dithiothreitol (Wako). Immediately after each treatment, the sperm suspension was centrifuged ( 5 min, $1400 \mathrm{~g}$ ) and cells and other components (supernatant) were separated. The supernatant was diluted in sample buffer and stored at $-20{ }^{\circ} \mathrm{C}$ until western blotting analysis.

\section{Collection of sperm tails}

Sperm tails were collected by density-gradient centrifugation as follows. Spermatozoa were washed three times in PBS. The pellet was then resuspended in PBS including protease inhibitors and sonicated for $10 \mathrm{~s}$. The sonicated sample was layered on a $150 \mu \mathrm{l}$ discontinuous gradient (consisting of $500 \mu \mathrm{l}$ layers of 40,60 , and $80 \%$ solutions respectively) of Percoll (GE Healthcare, Buckinghamshire, UK) in PBS supplemented with protease inhibitors, then subjected to centrifugation at $3200 \mathrm{~g}$ for $20 \mathrm{~min}$ at $4{ }^{\circ} \mathrm{C}$. After centrifugation, sperm tails had gathered in the $60 \%$ solution layer. To purify the sperm tails, the collected $60 \%$ solution was layered on the same type of Percoll gradient and centrifuged again. The tail fraction collected from the $60 \%$ solution layer after the second centrifugation was mixed with PBS supplemented with protease inhibitors and centrifuged at $9600 \mathrm{~g}$ for $10 \mathrm{~min}$ at $4{ }^{\circ} \mathrm{C}$. The pellets were finally resuspended in sample buffer and stored at $-20{ }^{\circ} \mathrm{C}$ until western blotting analysis.

\section{Oocyte collection and in vitro maturation}

Ovaries were obtained from prepubertal cross-bred gilts (Landrace, Large White, and Duroc breeds) at a local slaughterhouse and transported to the laboratory at $35^{\circ} \mathrm{C}$. Cumulus-oocyte complexes (COCs) were collected from follicles 2-6 mm in diameter in TCM 199 (with Hanks' salts; Sigma) supplemented with $10 \%(\mathrm{v} / \mathrm{v})$ fetal bovine serum (Gibco, Invitrogen), $20 \mathrm{mM}$ Hepes (Dojindo Laboratories), $100 \mathrm{IU} / \mathrm{ml}$ penicillin G potassium (Sigma), and $0.1 \mathrm{mg} / \mathrm{ml}$ streptomycin sulfate (Sigma). Maturation culture was performed as reported previously (Kikuchi et al. 2002). In brief, about 40 COCs were cultured in 4-well dishes (Nunclon Multidishes; Thermo Fisher Scientific, Inc., Roskilde, Denmark) for $20-22 \mathrm{~h}$ in $500 \mu \mathrm{l}$ maturation medium, a modified North Carolina State University (NCSU)-37 solution (Petters \& Wells 1993) containing $10 \%(\mathrm{v} / \mathrm{v})$ porcine follicular fluid, $0.6 \mathrm{mM}$ cysteine (Sigma), $50 \mu \mathrm{M} \beta$-mercaptoethanol (Sigma), $1 \mathrm{mM}$ dibutyl cAMP (dbcAMP; Sigma), 10 IU/ml eCG (PMS 1000 Tani $\mathrm{NZ}$; Nihon Zenyaku Kogyo, Koriyama, Japan), and $10 \mathrm{IU} / \mathrm{ml}$ hCG (Puberogen 1500 U; Sankyo, Tokyo, Japan). They were subsequently cultured for $24 \mathrm{~h}$ in maturation medium without $\mathrm{dbcAMP}$ and hormones. Maturation culture was carried out at $39{ }^{\circ} \mathrm{C}$ in an atmosphere of $\mathrm{CO}_{2}, \mathrm{O}_{2}$, and $\mathrm{N}_{2}$ adjusted to 5, 5, and $90 \%$ respectively. After maturation culture, cumulus cells were removed from the oocytes by treatment with $150 \mathrm{IU} / \mathrm{ml}$ hyaluronidase (Sigma) and gentle pipetting. Denuded oocytes with the first polar body were harvested under a stereomicroscope and used as in vitro matured oocytes.

\section{Procedure of sperm injection}

In total, two solutions were prepared for ICSI: 1) for oocytes, a modified NCSU-37 solution without glucose but supplemented with $0.17 \mathrm{mM}$ sodium pyruvate, $2.73 \mathrm{mM}$ sodium lactate, $4 \mathrm{mg} / \mathrm{ml}$ BSA, $50 \mu \mathrm{M} \beta$-mercaptoethanol (IVC-PyrLac; Kikuchi et al. 2002), and $20 \mathrm{mM}$ Hepes, with the osmolality adjusted to $285 \mathrm{mOsm} / \mathrm{kg}$ (IVC-PyrLac-Hepes; Nakai et al. 2003), and 2) for sperm, IVC-PyrLac-Hepes supplemented with $4 \%(\mathrm{w} / \mathrm{v})$ polyvinyl pyrrolidone (molecular weight 360 000; Sigma; IVC-PyrLac-Hepes-PVP). Spermatozoa were injected as described previously (Nakai et al. 2003). About 20 oocytes were transferred to a $20 \mu \mathrm{l}$ drop of IVC-PyrLac-Hepes. The solution containing the mature oocytes was placed on the cover of a plastic dish (Falcon 35-1005; Becton Dickinson and Company, Franklin Lakes, NJ, USA). A small volume $(0.5 \mu \mathrm{l})$ of the sperm suspension was transferred to a $2 \mu$ drop of IVCPyrLac-Hepes-PVP, which was prepared close to the drops used for the oocytes. All drops were covered with paraffin oil (Paraffin Liquid; Nakarai Tesque, Inc., Kyoto, Japan). The spermatozoa were injected into the ooplasms by using a Piezo-actuated micromanipulator (PMAS-CT150; Prime Tech Ltd, Tsuchiura, Japan). Sperm-injected oocytes were then cultured in IVC-PyrLac at $38.5^{\circ} \mathrm{C}$ under $5 \% \mathrm{O}_{2}$.

\section{Assessment of oocyte activation}

Injected oocytes were cultured for $10 \mathrm{~h}$ and then mounted on glass slides. The mounted oocytes were fixed in $25 \%(\mathrm{v} / \mathrm{v})$ acetic acid in ethanol and stained with $1 \%(\mathrm{w} / \mathrm{v})$ orcein in $45 \%$ $(\mathrm{v} / \mathrm{v})$ acetic acid. The nuclear status of the oocytes was observed using a phase-contrast microscope. We classified the states of the oocytes as follows: 1) 'normal fertilization' characterized by the presence of two PNs (male and female) and two polar bodies; 2) 'other $\mathrm{PN}^{\prime}$ ', including oocytes that had formed PN other than normal fertilized oocytes; 3) 'PN formation', including all oocytes with one or more $\mathrm{PN}(\mathrm{s})$, i.e. the total in categories 1 and 2; 4) 'transitional stage', in which oocytes had resumed meiosis but before any $\mathrm{PN}$ formation, i.e. total in anaphase-II, telophase-II, and metaphase-III (an abnormal stage in which chromosomes remain condensed even after telophase-Il due to insufficient ooplasmic activation); and 5) 'metaphase-II', in which oocytes were arrested at the metaphase-II stage. We considered categories (1-4) to be oocytes exhibiting activation phenomena. The assay was replicated at least three times using more than 50 oocytes per each injection group. 


\section{Western blotting}

Western blotting was carried out as described previously (Ito

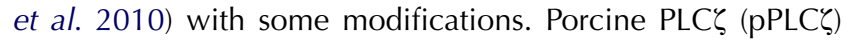
was detected using anti-PLC $\zeta$ rabbit serum generated against a 19-mer sequence (MENKWFLSMVRDDFKGGKI) at the $\mathrm{N}$-terminus of pPLC $\zeta$ (accession no. BAC78817) as described previously (Kurokawa et al. 2005). After denaturation by boiling at $99.5^{\circ} \mathrm{C}$ for $3 \mathrm{~min}$, samples were separated by SDS-PAGE on $12.5 \%$ polyacrylamide gel and then transferred onto a PVDF membrane (GE Healthcare). The membrane was blocked using blocking buffer (5\% (w/v) BSA in PBS) supplemented with $0.1 \%$ Tween 20 (T-PBS), then incubated with anti-PLC $\zeta$ antibody (1:5000) overnight at $4{ }^{\circ} \mathrm{C}$ in T-PBS. After three washes in T-PBS, the membranes were treated with HRP-labeled anti-rabbit IgG (1:5000, Cell Signaling Technology, Danvers, MA, USA) in blocking buffer for $1.5 \mathrm{~h}$ at room temperature. After one wash for $15 \mathrm{~min}$ and five washes for 5 min each with T-PBS, peroxidase activity was visualized using the ECL Plus western blotting detection system (GE Healthcare) in accordance with the manufacturer's instructions. The intensity of the bands was analyzed using ImageJ software (Ver. 1.41, National Institutes of Health, Bethesda, MD, USA).

\section{Immunofluorescence}

Immunofluorescence was performed as reported previously (Seita et al. 2009). Immediately after each treatment, sperm was centrifuged ( $600 \mathrm{~g}, 2 \mathrm{~min}$ ) and the pellet was resuspended and incubated with $3.7 \%$ paraformaldehyde in PBS for $30 \mathrm{~min}$ at $4{ }^{\circ} \mathrm{C}$. For permeabilization, $0.1 \%(\mathrm{v} / \mathrm{v}) \mathrm{TX}-\mathrm{PBS}$ was added to the samples and incubated for $10 \mathrm{~min}$ at room temperature. The sperm suspension was then spotted as $20 \mu \mathrm{l}$ drops onto glass slides and allowed to attach to the slides for $20 \mathrm{~min}$ at $37^{\circ} \mathrm{C}$. The slides were blocked in 5\% normal goat serum (NGS, Cedarlane Laboratories Ltd, Hornby, ON, Canada) in PBS for $3 \mathrm{~h}$ at $4{ }^{\circ} \mathrm{C}$ and then incubated overnight at $4{ }^{\circ} \mathrm{C}$ with anti$\operatorname{pPLC} \zeta(1: 200)$ in $5 \%$ NGS. Washes were performed with T-PBS, followed by $1 \mathrm{~h}$ incubation at room temperature with Alexa Fluor 488-labeled goat anti-rabbit antibody (1:200; Molecular Probes, Inc., Eugene, OR, USA) as the secondary antibody. After several washings in T-PBS, samples were counterstained with propidium iodide (Molecular Probes). Fluorescence images were obtained using a fluorescence microscope (IX-71; Olympus, Tokyo, Japan). As a negative control, sperm were immunostained using the protocol described above, but without the primary antibody. We also confirmed the specificity of pig PLC $\zeta$ using an antigenic peptide for PLC $\zeta 10 \mathrm{ng} / \mu \mathrm{l}$.

\section{Statistical analysis}

Statistical analysis of the data from more than three replicates was carried out by ANOVA and Tukey's multiple range tests (Statistical Analysis System (SAS, ver. 9.2; SAS Institute, Inc., Cary, NC, USA). All percentage data were subjected to arcsine transformation (Snedecor \& Cochran 1989) before statistical analysis. Differences were considered significant at $P<0.05$.

\section{Declaration of interest}

The authors declare that there is no conflict of interest that could be perceived as prejudicing the impartiality of the research reported.

\section{Funding}

This research was supported by the Japanese Society for Promotion of Science (JSPS) Fellowship (71310042 to M Nakai), Grant-in-Aid for Scientific Research (21789253 to J Ito, 22658085 to N Kashiwazaki, 21380715 to H Kaneko, and 22380153 to K Kikuchi) from JSPS, the Promotion and Mutual Aid Corporation for Private Schools of Japan through a Grantin-Aid for Matching Fund Subsidy for Private Universities to $\mathrm{J}$ Ito and N Kashiwazaki and a project grant (Creative Research Project, 2010) awarded by the Azabu University Research Services Division.

\section{Acknowledgements}

The authors would like to thank Prof. Rafael A Fissore, Department of Veterinary and Animal Sciences, University of Massachusetts, for reviewing this manuscript. We would also like to thank Drs Nagai T, Onishi A, Somfai T, Ozawa M, Maedomari N, Fahrudin M and Karja NWK for completion of experiments on this study and also Mss Kaneko K, Osaki M, and Aoki $\mathrm{T}$ for technical assistance.

\section{References}

Bedford-Guaus SJ, McPartlin LA, Westmiller SL, Buffone MG \& Roberson MS 2011 Molecular cloning and characterization of phospholipase $\mathrm{C}$ zeta in equine sperm and testis reveals species-specific differences in expression of catalycally active protein. Biology of Reproduction 85 78-88. (doi:10.1095/biolreprod.110.089466)

Bos-Mikich A, Whittingham DG \& Jones KT 1997 Meiotic and mitotic $\mathrm{Ca}^{2+}$ oscillations affect cell composition in resulting blastocysts. Developmental Biology 182 172-179. (doi:10.1006/dbio.1996.8468)

Catt SL, Catt JW, Gomez MC, Maxwell WMC \& Evans G 1996 The birth of a male lamb derived from an in vitro matured oocyte fertilized by intracytoplasmic injection of a single presumptive male sperm. Veterinary Record 139 494-495. (doi:10.1136/vr.139.20.494)

Cochran R, Meintjes M, Reggio B, Hylan D, Carter J, Pinto C, Paccamonti D \& Godke RA 1998 Live foals produced from sperminjected oocytes derived from pregnant mares. Journal of Equine Veterinary Science 18 736-740. (doi:10.1016/S0737-0806(98)80504-2)

Coward K, Ponting CP, Chang HY, Hibbitt O, Savolainen P, Jones KT \& Parrington J 2005 Phospholipase Czeta, the trigger of egg activation in mammals, is present in a non-mammalian species. Reproduction 130 157-163. (doi:10.1530/rep.1.00707)

Cox LJ, Larman MG, Saunders CM, Hashimoto K, Swann K \& Lai FA 2002 Sperm phospholipase Czeta from humans and cynomolgus monkeys triggers $\mathrm{Ca}^{2+}$ oscillations, activation and development of mouse oocytes. Reproduction 124 611-623. (doi:10.1530/rep.0.1240611)

Ducibella T, Huneau D, Angelichio E, Xu Z, Schultz RM, Kopf GS, Fissore R, Madoux S \& Ozil JP 2002 Egg-to-embryo transition is deriven by differential responses to $\mathrm{Ca}^{2+}$ oscillation number. Developmental Biology 250 280-291. (doi:10.1006/dbio.2002.0788)

Fujinami N, Hosoi Y, Kato H, Matsumoto K, Saeki K \& Iritani A 2004 Activation with ethanol improves embryo development of ICSI-derived oocytes by regulation of kinetics of MPF activity. Journal of Reproduction and Development 50 171-178. (doi:10.1262/jrd.50.171) 
Galli C, Vassiliev I, Lagutina I, Galli A \& Lazzari G 2003 Bovine embryo development following ICSI: effect of activation, sperm capacitation and pre-treatment with dithiothereitol. Theriogenology 60 1467-1480. (doi:10.1016/S0093-691X(03)00133-X)

Garcia-Rosello E, Garcia-Mengual E, Coy P, Alfonso J \& Silvestre MA 2009 Intracytoplasmic sperm injection in live stock species: an update. Reproduction in Domestic Animals 44 143-151. (doi:10.1111/j.14390531.2007.01018.x)

Goto K, Kinoshita A, Takuma Y \& Ogawa K 1990 Fertilization of bovine oocytes by the injection of immobilized, killed spermatozoa. Veterinary Record 127 517-520. (doi:10.1136/vr.127.21.517)

Gou KM, An XR, Tian JH \& Chen YF 2002 Sheep transgenic embryos produced by intracytoplasmic sperm injection. Shi Yan Sheng $W u$ Xue Ваo 35 103-108.

Heytens E, Parrington J, Conward K, Young C, Lambrecht S, Yoon SY, Fissore RA, Hamer R, Deane CM, Ruas M et al. 2009 Reduced amounts and abnormal forms of phospholipase Czeta (PLC $\zeta$ ) in spermatozoa from infertile men. Human Reproduction 24 2417-2428. (doi:10.1093/ humrep/dep207)

Hirabayashi M, Kato M, Aoto T, Sekimoto A, Ueda M, Miyoshi I, Kasai N \& Hochi S 2002 Offspring derived from intracytoplasmic injection of transgenic rat sperm. Transgenic Research 11 221-228. (doi:10.1023/ A:1015210604906)

Ikeda H, Kikuchi K, Noguchi J, Takeda H, Shimada A, Mizokami T \& Kaneko H 2002 Effect of preincubation of cryopreserved porcine epididymal sperm. Theriogenology 57 1309-1318. (doi:10.1016/ S0093-691X(02)00630-1)

Ito M, Shikano T, Oda S, Horiuchi T, Tanimoto S, Awaji T, Mitani H \& Miyazaki S 2008 Difference in $\mathrm{Ca}^{2+}$ oscillations-inducing activity and nuclear translocation ability of PLCZ1, an egg-activating sperm factor candidate, between mouse, rat, human and medaka fish. Biology of Reproduction 78 1081-1090. (doi:10.1095/biolreprod.108.067801)

Ito J, Yoshida T, Kasai Y, Wakai T, Parys JB, Fissore RA \& Kashiwazaki N 2010 Phosphorylation of inositol 1,4,5-triphosphate receptor 1 during in vitro maturation of porcine oocytes. Animal Science Journal 81 34-41. (doi:10.1111/j.1740-0929.2009.00699.x)

Jimenez-Macedo AR, Izquierdo D, Anquita B \& Paramio MT 2005 Comparison between intracytoplasmic sperm injection and in vitro fertilization employing oocytes derived from prepubertal goats. Theriogenology 64 1249-1262. (doi:10.1016/j.theriogenology.2004. 11.025)

Katayama M, Miyano T, Miyake M \& Kato S 2002 Progesterone treatment of boar spermatozoa improves male pronuclear formation after intracytoplasmic sperm injection into porcine oocytes. Zygote 10 95-104. (doi:10.1017/S0967199402002137)

Katayama M, Sutovsky P, Yang BS, Cantley T, Rieke A, Farwell R, Oko R \& Day BN 2005 Increased disruption of sperm plasma membrane at sperm immobilization promotes dissociation of perinuclear theca from sperm chromatin after intracytoplasmic sperm injection in pigs. Reproduction 130 907-916. (doi:10.1530/rep.1.0680)

Kikuchi K, Nagai T, Ikeda H, Noguchi J, Shimada A, Soloy E \& Kaneko H 1998 Cryopreservation and ensuing in vitro fertilization ability of boar spermatozoa from epididymies stored at $4{ }^{\circ} \mathrm{C}$. Theriogenology $\mathbf{5 0}$ 615-623. (doi:10.1016/S0093-691X(98)00166-6)

Kikuchi K, Kashiwazaki N, Noguchi J, Shimada A, Takahashi R, Hirabayashi M, Shino M, Ueda M \& Kaneko H 1999 Developmental competence, after transfer to recipients, of porcine oocytes matured, fertilized, and cultured in vitro. Biology of Reproduction 60 336-340. (doi:10.1095/biolreprod60.2.336)

Kikuchi K, Onishi A, Kashiwazaki N, Iwamoto M, Noguchi J, Kaneko H, Akita T \& Nagai T 2002 Successful piglet production after transfer of blastocysts produced by a modified in vitro system. Biology of Reproduction 66 1033-1041. (doi:10.1095/biolreprod66.4.1033)

Kim NH, Jun SH, Do JT, Uhm SJ, Lee HT \& Chung KS 1999 Intracytoplasmic injection of porcine, bovine, mouse, or human spermatozoon into porcine oocytes. Molecular Reproduction and Development 53 84-91. (doi:10.1002/(SICl)1098-2795(199905)53:1 < 84::AID-MRD10>3.0. $\mathrm{CO} ; 2-\mathrm{U})$

Kimura Y \& Yanagimachi R 1995 Intracytoplasmic sperm injection in the mouse. Biology of Reproduction 52 709-720. (doi:10.1095/ biolreprod52.4.709)
Kolbe T \& Holtz W 1999 Intracytoplasmic injection (ICSI) of in vivo or in vitro matured oocytes with fresh ejaculated or frozen-thawed epididymal spermatozoa and additional calcium-ionophore activation in the pig. Theriogenology 52 671-682. (doi:10.1016/S0093-691X(99) 00161-2)

Kolbe T \& Holtz W 2000 Birth of a piglet derived from an oocyte fertilized by intracytoplasmic sperm injection (ICSI). Animal Reproduction Science 64 97-101. (doi:10.1016/S0378-4320(00)00204-9)

Kren R, Kikuchi K, Nakai M, Miyano T, Ogushi S, Nagai T, Suzuki S, Fulka J \& Fulka J Jr 2003 Intracytoplasmic sperm injection in the pig: where is the problem? Journal of Reproduction and Development 49 271-273. (doi:10.1262/jrd.49.271)

Kuretake S, Kimura Y, Hoshi K \& Yanagimachi R 1996 Fertilization and development of mouse oocytes injected sperm head. Biology of Reproduction 55 789-795. (doi:10.1095/biolreprod55.4.789)

Kurokawa M, Sato K, Wu H, He C, Malcuit C, Black SJ, Fukami K \& Fissore RA 2005 Functional, biochemical, and chromatographic characterization of the complete $[\mathrm{Ca} 2+]$ oscillation-inducing activity of porcine sperm. Developmental Biology 285 376-392. (doi:10.1016/j. ydbio.2005.06.029)

Lee JW \& Yang X 2004 Factors affecting fertilization of porcine oocytes following intracytoplasmic injection of sperm. Molecular Reproduction and Development 68 96-102. (doi:10.1002/mrd.20044)

Lee JW, Tian XC \& Yang X 2003 Failure of male pronucleus formation is the major cause of lack of fertilization and embryo development in pig oocytes subjected to intracytoplasmic sperm injection. Biology of Reproduction 68 1341-1347. (doi:10.1095/biolreprod.102.009001)

Martin MJ 2000 Development of in vivo-matured porcine oocytes following intracytoplasmic sperm injection. Biology of Reproduction 63 109-112. (doi:10.1095/biolreprod63.1.109)

Miyazaki S \& Ito M 2006 Calcium signals for egg activation in mammals. Journal of Pharmacological Sciences 100 545-552. (doi:10.1254/jphs. CPJ06003X)

Mizushima S, Takagi S, Ono T, Atsumi Y, Tsukada A, Saito N \& Shimada K 2009 Phospholipase $C \zeta$ mRNA expression and its potency during spermatogenesis for activation of quail oocyte as a sperm factor. Molecular Reproduction and Development 76 1200-1207. (doi:10. 1002/mrd.21097)

Nagai T 1994 Current status and perspectives in IVM-IVF of porcine oocytes. Theriogenology $\mathbf{4 1}$ 73-78. (doi:10.1016/S0093-691X(05) 80051-2)

Nakai M, Kashiwazaki N, Takizawa A, Hayashi Y, Nakatsukasa E, Fuchimoto D, Noguchi J, Kaneko H, Shino M \& Kikuchi K 2003 Viable piglets generated from porcine oocytes matured in vitro and fertilized by intracytoplasmic sperm head injection. Biology of Reproduction 68 1003-1008. (doi:10.1095/biolreprod.102.009506)

Nakai M, Saito E, Takizawa A, Akamatsu Y, Koichi M, Hisamatsu S, Inomata T, Shino M \& Kashiwazaki N 2005 Offspring derived from intracytoplasmic injection of isolated sperm heads. Journal of Mammalian Ova Research 22 159-162. (doi:10.1274/jmor.22.159)

Nakai M, Kashiwazaki N, Takizawa A, Maedomari N, Ozawa M, Noguchi J, Kaneko H, Shino M \& Kikuchi K 2006 Morphological changes in boar sperm nuclei with reduced disulfide bonds in electrostimulated porcine oocytes. Reproduction 131 603-611. (doi:10.1530/rep.1.01001)

Ozil JP, Banrezes B, Toth S, Pan H \& Schultz RM $2006 \mathrm{Ca}^{2+}$ oscillatory pattern in fertilized mouse eggs affects gene expression and development to term. Developmental Biology 300 534-544. (doi:10.1016/j.ydbio. 2006.08.041)

Petters RM \& Wells KD 1993 Culture of pig embryos. Journal of Reproduction and Fertility. Supplement $\mathbf{4 8}$ 61-73.

Probst S \& Rath D 2003 Production of piglets using intracytoplasmic sperm injection (ICSI) with flowcytometrically sorted boar semen and artificially activated oocytes. Theriogenology 59 961-973. (doi:10.1016/S0093691X(02)01135-4)

Ross PJ, Beyhan Z, lager AE, Yoon SK, Malcuit C, Schellander K, Fissore RA \& Cibelli JB 2008 Parthenogenetic activation of bovine oocytes using bovine and murine phospholipase Czeta. BMC Developmental Biology 8 16. (doi:10.1186/1471-213X-8-16)

Saunders CM, Larman MG, Parrington J, Cox LJ, Royse J, Blayney LM, Swann K \& Lai FA 2002 PLC : a sperm-specific trigger of $\mathrm{Ca}^{2+}$ oscillations in eggs and embryo development. Development $1293533-3544$. 
Seita Y, Ito J \& Kashiwazaki N 2009 Removal of acrosomal membrane from sperm head improves development of rat zygotes derived from intracytoplasmic sperm injection. Journal of Reproduction and Development 55 475-479. (doi:10.1262/jrd.20216)

Snedecor GW \& Cochran WG 1989 Statistical Methods, edn 8, pp 273-296. Ames, IA: lowa State University Press.

Suzuki K, Asano A, Eriksson B, Niwa K, Nagai T \& Rodriguez-Martinez H 2002 Capacitation atatus and in vitro fertility of boar spermatozoa: effects of seminalplasma, cumulus-oocyte-complexes-conditioned medium and hyaluronan. International Journal of Andrology 25 84-93. (doi:10. 1046/j.1365-2605.2002.00330.x)

Yamauchi Y, Yanagimachi R \& Horiuchi T 2002 Full-term development of gorden hamster oocytes following intracytoplasmic sperm head injection. Biology of Reproduction 67 534-539. (doi:10.1095/biolreprod67.2.534)

Yanagimachi R 2005 Intracytoplasmic injection of spermatozoa and spermatogenic cells: its biology and applications in humans and animals. Reproductive Biomedicine Online 10 247-288. (doi:10.1016/S14726483(10)60947-9)

Yazawa H, Yanagida K, Hayashi S \& Sato A 2009 The oocyte activation and $\mathrm{Ca}^{2+}$ oscillation-inducing abilities of mouse and human dead (sonicated) spermatozoa. Zygote 17 175-184. (doi:10.1017/S0967199408005157)

Yoneda A, Kashima M, Yoshida S, Terada K, Nakagawa S, Sakamoto A, Hayakawa K, Suzuki K, Ueda J \& Watanabe T 2006 Molecular cloning, testicular postnatal expression, and oocyte-activating potential of porcine phospholipase Czeta. Reproduction 132 393-401. (doi:10. 1530/rep.1.01018)
Yong HY, Hong JY, Kang SK, Lee BC, Lee ES \& Hwang WS 2005 Sperm movement in the ooplasm, dithiothreitol pretreatment and sperm freezing are not required for the development of porcine embryos derived from injection of head membrane-damaged sperm. Theriogenology 63 783-794. (doi:10.1016/j.theriogenology.2004.05.004)

Yoon SY \& Fissore RA 2007 Release of phospholipase C $\zeta$ and $[\mathrm{Ca} 2+] \mathrm{i}$ oscillation-inducing activity during mammalian fertilization. Reproduction 134 695-704. (doi:10.1530/REP-07-0259)

Yoon SY, Jellerette T, Salicioni AM, Lee HC, Yoo MS, Coward K, Parrington J, Grow D, Cibelli JB, Visconti PE et al. 2008 Human sperm devoid of PLC, zeta1 fail to induce $\mathrm{Ca}^{2+}$ release and are unable to initiate the first step of embryo development. Journal of Clinical Investigation 118 3671-3681. (doi:10.1172/JCI36942)

Young C, Grasa P, Conward K, Davis LC \& Parrington J 2009 Phospholipase Czeta undergoes dynamic changes in its pattern of localization in sperm during capacitation and the acrosome reaction. Fertility and Sterility 91 2230-2242. (doi:10.1016/j.fertnstert. 2008.05.021)

Received 9 March 2011

First decision 7 April 2011

Revised manuscript received 13 May 2011

Accepted 24 May 2011 